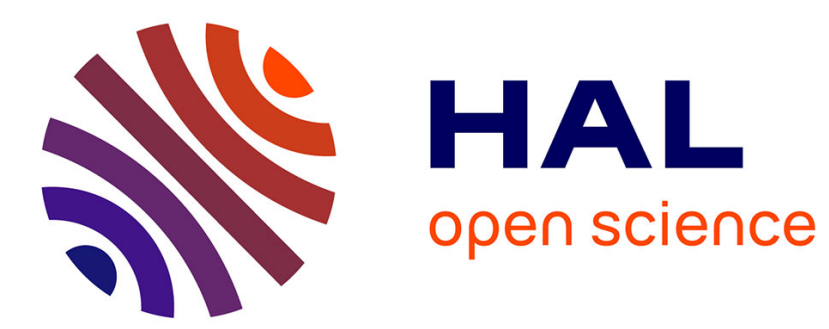

\title{
Nonlinear gyrofluid simulations of collisionless reconnection
}

D. Grasso, Emanuele Tassi, F. Waelbroeck

\section{To cite this version:}

D. Grasso, Emanuele Tassi, F. Waelbroeck. Nonlinear gyrofluid simulations of collisionless reconnection. Physics of Plasmas, 2010, 17 (8), pp.082312. 10.1063/1.3475440 . hal-00518375

\section{HAL Id: hal-00518375 \\ https://hal.science/hal-00518375}

Submitted on 17 Sep 2010

HAL is a multi-disciplinary open access archive for the deposit and dissemination of scientific research documents, whether they are published or not. The documents may come from teaching and research institutions in France or abroad, or from public or private research centers.
L'archive ouverte pluridisciplinaire HAL, est destinée au dépôt et à la diffusion de documents scientifiques de niveau recherche, publiés ou non, émanant des établissements d'enseignement et de recherche français ou étrangers, des laboratoires publics ou privés. 


\title{
Nonlinear gyrofluid simulations of collisionless reconnection
}

\author{
D. Grasso ${ }^{1}$, E. Tassi ${ }^{2}$, F.L. Waelbroeck ${ }^{3}$ \\ ${ }^{1}$ CNR-INFM, Dipartimento di Energetica, Politecnico di Torino, \\ Corso Duca degli Abruzzi, 24, 10129, Torino, Italy \\ 2 Centre de Physique Théorique, CNRS - Aix-Marseille Universités, \\ Campus de Luminy, case 907, F-13288 Marseille cedex 09, France \\ ${ }^{3}$ Institute for Fusion Studies, The University of Texas at Austin, Austin, TX 78712-1060, USA
}

\begin{abstract}
The Hamiltonian gyrofluid model recently derived by Waelbroeck et al. [Phys. Plasmas 16, 032109 (2009)], is used to investigate nonlinear collisionless reconnection with a strong guide field by means of numerical simulations. Finite ion Larmor radius gives rise to a cascade of the electrostatic potential to scales below both the ion gyroradius and the electron skin depth. This cascade is similar to that observed previously for the density and current in models with cold ions. In addition to density cavities, the cascades create electron beams at scales below the ion gyroradius. The presence of finite ion temperature is seen to modify, inside the magnetic island, the distribution of the velocity fields that advect two Lagrangian invariants of the system. As a consequence, the fine structure in the electron density is confined to a layer surrounding the separatrix. Finite ion Larmor radius effects produce also a different partition between the electron thermal, potential, and kinetic energy, with respect to the cold ion case. Other aspects of the dynamics such as the reconnection rate and the stability against Kelvin-Helmholtz modes, are similar to simulations with finite electron compressibility but cold ions.
\end{abstract}

\section{INTRODUCTION}

The investigation of collisionless magnetic reconnection by means of Hamiltonian reduced fluid models has proved to be useful in various ways, for instance in the interpretation of nonlinear structures observed in simulations [1-6], the derivation of stability criteria [7], the extension of the model to include external fields while preserving a Hamiltonian structure [6], and the identification of negative energy modes [7]. The above mentioned results refer, however, to cold-ion Hamiltonian models. Energy-conserving, hot-ion (in particular, gyrofluid) versions of such cold-ion models have been derived, for instance in Refs.[8-12]. In particular, a gyrofluid two-field version of the model presented in Ref.[9] was investigated numerically in Ref.[13] and a dissipative version of it was studied in Ref. [14]. A separate application of this model to the study of Alfvénic turbulence appeared after completion of the present work.[15]

Although different energy-conserving, hot-ion models are available, the nonlinear numerical investigation of Hamiltonian gyrofluid models for collisionless reconnection is relatively less developed with respect to that in the cold-ion limit. An example of results in this direction was presented in Ref. [13]. The analysis presented in that article, however, was limited to the linear and early nonlinear phase of the dynamics. The purpose of this article is to investigate the nonlinear dynamics of a Hamiltonian gyrofluid model and compare the results with those obtained in Ref.[13] and in previous investigations of cold-ion models. Particular emphasis will be given to the analysis of the structures that form nonlinearly in the fields. The structure of the electric field, for example, is of interest in theories 
of electron [16, 17] and ion [18] energization during magnetic reconnection in the magnetopause, the solar corona, and in laboratory experiment $[19,20]$. Note, however, that energization is a kinetic process that cannot be described rigorously within the fluid model used here. We will investigate, with the help of the Hamiltonian structure of the model, the role played by the finite ion Larmor radius on the electron density distribution. The influence of finite ion temperature on the secondary fluid instabilities observed in recent years $[4,5,21,22]$ will also be considered.

The paper is organized as follows: in Sec. II the model equations are introduced. Sec. III and Sec. IV are devoted to the analysis of the results of the numerical simulations, with focus on field structures and energy partition, respectively. Conclusions are drawn in Sec. V.

\section{MODEL EQUATIONS}

We consider the following Padé approximant, two-dimensional version of the Hamiltonian gyrofluid model of Ref.[12]:

$$
\begin{array}{r}
\frac{\partial n_{i}}{\partial t}+\left[\Phi, n_{i}\right]=0, \\
\frac{\partial n_{e}}{\partial t}+\left[\phi, n_{e}\right]-\left[\psi, \nabla^{2} \psi\right]=0, \\
\frac{\partial}{\partial t}\left(\psi-d_{e}^{2} \nabla^{2} \psi\right)+\left[\phi, \psi-d_{e}^{2} \nabla^{2} \psi\right]+\rho_{s}^{2}\left[\psi, n_{e}\right]=0, \\
n_{e}=\frac{1}{1-\frac{\rho_{i}^{2}}{2} \nabla^{2}} n_{i}+\frac{\nabla^{2}}{1-\rho_{i}^{2} \nabla^{2}} \phi .
\end{array}
$$

Given a Cartesian coordinate system $(x, y, z)$, we assume that all the fields are translationally invariant along $z$. The variables in Eqs.(1)-(4) are written in a dimensionless form and their relations with dimensional quantities are given by:

$$
\begin{array}{r}
t=\frac{v_{A}}{L} \hat{t}, \quad x=\frac{\hat{x}}{L}, \quad n_{i}=\frac{L}{\hat{d}_{i}} \frac{\hat{n}_{i}}{n_{0}}, \\
d_{e}=\frac{\hat{d}_{e}}{L}, \quad n_{e}=\frac{L}{\hat{d}_{i}} \frac{\hat{n}_{e}}{n_{0}}, \quad \psi=\frac{\hat{A}_{z}}{B L}, \quad \phi=\frac{\hat{\rho}_{s}^{2}}{L^{2}} \frac{L}{\hat{d}_{i}} \frac{e \hat{\phi}}{T_{e}},
\end{array}
$$

where the carets denote dimensional variables. In $(5) n_{0}$ indicates a background density, $L$ is a characteristic magnetic equilibrium scale length, $v_{A}$ is the Alfvèn speed based on a characteristic poloidal magnetic field intensity $B, \hat{n}_{i, e}$ represent the ion guiding center and the electron density, respectively, $\hat{A}_{z}$ is the $z$ component of the vector potential, $\hat{\rho}_{s}$ is the sonic Larmor radius, $\hat{\phi}$ is the electrostatic potential, $\hat{d}_{i, e}$ the ion and electron skin depth, $T_{i, e}$ the ion and electron temperature, respectively, and $e$ is the unit charge. In addition to this, we indicate with $\rho_{i}=\sqrt{T_{i} / T_{e}} \rho_{s}$ the 
ion Larmor radius, and with

$$
\Phi=\frac{1}{1-\frac{\rho_{i}^{2}}{2} \nabla^{2}} \phi
$$

the gyroaveraged electrostatic potential. Note that this normalization differs from that adopted in Ref. [12].

It is easy to see that in the limit $\rho_{i} \rightarrow 0$, the ions decouple and the above equations effectively reduce to the twofield, cold-ion model used in [2, 23]. This model as well as the more complete model used here, describe the so-called "inertial-Alfvén" regime in the limit $\beta_{e} \ll m_{e} / m_{i}$ (or equivalently, $\rho_{s} \ll d_{e}$ ) as well as the "kinetic Alfvén" regime in the opposite limit. In the previous expression, we indicated with $m_{e, i}$ the electron and ion mass, respectively, and with $\beta_{e}$ the ratio between the electron pressure and the magnetic pressure. These two regimes have been observed in laboratory experiments [24] as well as in the auroral region with the Freja satellite. [25] The characteristics of magnetic reconnection in the two limits $\beta_{e}<m_{e} / m_{i}$ and $\beta_{e}>m_{e} / m_{i}$ have been compared and contrasted by Rogers et al. [26]

\section{ANALYSIS OF THE FIELD STRUCTURES}

We perform numerical simulations of the model (1)-(4) on the domain $\{(x, y):-\pi \leq x<\pi,-\pi \leq y<\pi\}$, with a grid of $1024 \times 128$ points and imposing double periodic boundary conditions. The initial equilibrium is given by

$$
n_{i_{e q}}(x)=n_{0}, \quad n_{e_{e q}}(x)=n_{0}, \quad \psi_{e q}(x)=\sum_{n=-11}^{11} a_{n} \exp (i n x)
$$

where $n_{0}$ is a constant background density and the $a_{n}$ are the Fourier coefficients of the function $f(x)=1 / \cosh ^{2} x$. In Ref. [27] it has been shown that such truncated Fourier series provides a very good representation of the equilibrium flux function. The equilibrium of the form $1 / \cosh ^{2} x$ makes it possible to avoid the early cross-talking between magnetic islands that prevented the observation of a developed nonlinear phase in Ref.[13]. The equilibrium (7) allows to reach a nonlinear phase, in which the ratio between the island width and the electron skin depth is approximately twice as much as that permitted in Ref.[13], and eventually a saturation phase. The equilibrium is destabilized by perturbing the $n_{i}$ field with a four-cell pattern disturbance of the form $\tilde{n}_{i} \propto \cos (x+y)-\cos (x-y)$. The field $\phi$ is perturbed accordingly, in such a way that the initial perturbation on $n_{e}$ is zero. The choice (7) for the equilibrium implies that $n_{i}$ grows only very weakly compared to the other fields. Although this choice makes the role of $n_{i}$ on the dynamics essentially negligible, it permits to make a more direct comparison with previous results. An investigation in the presence of an ion guiding center nonuniform equilibrium will be the subject of a future publication. 
Simulations have been run fixing $d_{e}=0.2$ and varying the values of $\rho_{s}$ and $\rho_{i}$, in order to investigate the influence on the dynamics of finite ion temperature with respect to cold ion models or to the gyrofluid model of Ref. [13]. Linear growth rates observed in the simulations have been compared with the asymptotic formula $\gamma \approx 2\left(2 d_{e} \rho_{\tau}^{2} / \pi\right)^{1 / 3}$, derived from linear theory [28], and a good agreement was found (here $\rho_{\tau}^{2}=\rho_{i}^{2}+\rho_{s}^{2}$ and the multiplicative factor 2 comes from the choice of the magnetic equilibrium adopted here).

We first carried out a comparison between simulations obtained by setting alternatively $\rho_{s}$ and $\rho_{i}$ equal to 0.4 and 0.01. The case $\rho_{i}=0.4, \rho_{s}=0.01$, although unphysical, makes it possible to observe and isolate the effect of ion temperature on the cold plasma state. Results are shown in Fig. 1. Because the lengths of the initial transient phases are different in the two cases, we did not compare the fields at the same time but rather when the magnetic island has reached approximately the same width, which indicates that the two dynamics have reached the same degree of advancement.

From comparing the plots of the electron density one can see that the structures observed in the two simulations are qualitatively similar, in particular in the lobes around the magnetic separatrices. Such structures have already been observed in previous nonlinear simulations of different cold ion models (e.g. [2, 4, 6]). The inner regions look somewhat different, with a more accentuated quadrupolar structure in the $\rho_{s}=0.4$ case.

In order to understand the observed behavior we note the following: let us assume that the contribution coming from $n_{i}$ is negligible in (4). One can indeed verify that the amplitude of such term is typically much smaller than that of the other terms in the equation, namely due to the homogeneity of $n_{i}$ in the initial state. We then have the relation

$$
\phi=\nabla^{-2} n_{e}-\rho_{i}^{2} n_{e}
$$

Through this relation we can eliminate $\phi$ in (2)-(3) and obtain

$$
\begin{gathered}
\frac{\partial n_{e}}{\partial t}+\left[\nabla^{-2} n_{e}, n_{e}\right]-\left[\psi, \nabla^{2} \psi\right]=0 \\
\frac{\partial}{\partial t}\left(\psi-d_{e}^{2} \nabla^{2} \psi\right)+\left[\nabla^{-2} n_{e}, \psi-d_{e}^{2} \nabla^{2} \psi\right]+\left(\rho_{s}^{2}+\rho_{i}^{2}\right)\left[\psi, n_{e}\right]+\rho_{i}^{2} d_{e}^{2}\left[n_{e}, \nabla^{2} \psi\right]=0 .
\end{gathered}
$$

We can now see that in the evolution equation for $n_{e}(9)$ there is no explicit dependence on $\rho_{i}$ and $\rho_{s}$. These parameters appear explicitly only in the electron momentum equation (10). Note, however, that the coefficient of $\left[\psi, n_{e}\right]$ in $(10)$, takes the same value in the two cases of our simulations. Therefore, apart from the term $\rho_{i}^{2} d_{e}^{2}\left[n_{e}, \nabla^{2} \psi\right]$, the two 
systems we solve in the simulations are very similar. Thus, when it comes to determining the reconnection rate and qualitative field structures, interchanging the role of $\rho_{i}$ and $\rho_{s}$ can be expected to produce little differences, as already observed in Ref. [13]. This is reminiscent of the case with zero guide-field investigated in the "GEM challenge." In that case a comparison of kinetic, hybrid, and fluid (cold ion) codes showed that the reconnection rate is insensitive to the details of the plasma dynamics. [29] Subsequent work found that for all the models investigated in the GEM challenge, fast reconnection occurs when the phase velocity of the wave mediating the reconnection increases with the wavevector in a certain range. [26] Note, however, that more recent studies of reconnection in electron-positron plasmas have cast doubt on the role of the dispersion properties on the reconnection rate (see [30] and references therein).

In the case of the model considered here, when considering homogeneous equilibria $n_{i_{e q}}=n_{0}, n_{e_{e q}}=n_{0}, \psi_{e q}(x)=\alpha_{\psi} x$, with constant $\alpha_{\psi}$, the linear dispersion relation resulting from Eqs. (1)-(4) reads

$$
\omega= \pm\left|\alpha_{\psi} k_{y}\right| \sqrt{\frac{1+\left(\rho_{i}^{2}+\rho_{s}^{2}\right) k_{\perp}^{2}}{1+d_{e}^{2} k_{\perp}^{2}}} .
$$

In Eq. (11) $\omega$ is the frequency of propagation of the wave, $k_{y}$ is the wave vector along the $y$ direction and $k_{\perp}$ is the perpendicular wave vector. Note that this dispersion relation includes the so-called "inertial-Alfvén" regime in the limit $\beta_{e} \ll m_{e} / m_{i}$ or equivalently, $\rho_{s} \ll d_{e}$, as well as the "kinetic Alfvén" regime in the opposite limit. [24, 25] The expression (11) indicates that the inclusion of finite ion temperature does not modify the dispersive properties of the wave, with respect to the $\rho_{i}=0, \rho_{s} \neq 0$ case. It simply amounts to introducing an effective sonic radius $\rho_{\tau}=\sqrt{\rho_{i}^{2}+\rho_{s}^{2}}$. In this respect, the cold plasma case is qualitatively different.

Another similarity between the effect of the ion and electron temperature is that the secondary Kelvin-Helmholtz instability, observed in cold plasma models, $[4,5,21,22]$ is suppressed when $\rho_{i} \neq 0$, even if $\rho_{s}$ is very small. Indeed, even in the cold electron case, if finite ion temperature is taken into account, the formation of thinning layers prone to the fluid instability is suppressed by the appearance of lobes enclosed in the separatrices. We can then conclude that finite temperature effects, of either the electron or of the ion species, inhibit the onset of a secondary Kelvin-Helmholtz instability.

The similarities in the growth rates, electron density structures around the separatrices and Kelvin-Helmholtz stability properties, however, should not be allowed to distract from an important qualitative difference between the cold-ion and hot-ion cases: for hot ions, the electrostatic potential develops very fine structure, whereas for cold ions, it varies no faster than on the scale of $\rho_{s}$. This difference, which is clearly visible in Fig. 1, is confirmed by comparing 
profiles of $|\nabla \phi|$ in Fig. 2. Such plots indeed show that, as $\rho_{i}$ increases, strong electric fields develop in the plane perpendicular to the guide field, in regions surrounding the separatrices. In order to highlight this aspect, we chose to show the profiles at the chord $y / \pi=-1 / 2$, which crosses such regions. Formation of fine structures and steep gradients in the electric field accompany this growth in amplitude. Evidently, the same trend can be read also in terms of an increase in amplitude and in gradients of the $\mathbf{E} \times \mathbf{B}$ drift. The fine structure of the electrostatic potential influences also the parallel electric field. Since $E_{\|}=-\partial \psi / \partial t-[\phi, \psi]$, the latter field too develops small scales as $\rho_{i}$ increases. As an example, the contour plot of $E_{\|}$is drawn in fig. 3 for the case with $\rho_{i}=\rho_{s}=0.4$. Fig. 3 shows clearly that all the area enclosed by the magnetic island is dominated by fine structures. This behavior of the parallel electric field, combined with the formation of density cavities, is suggestive [16] of a possible enhancement of the particle acceleration along magnetic field line and, consequently, of the plasma heating as $\rho_{i}$ is increased.

To understand the reason for fine-scale structure of $\phi$, when $\rho_{s}$ is large, it is necessary to recall the conservation properties of the system. The study of these conservation properties $[2,12]$ leads to the conclusion that the dynamical equations (1)-(3) can be cast in the form of the following set of convection equations:

$$
\frac{\partial G_{j}}{\partial t}+\mathbf{v}_{j} \cdot \nabla G_{j}=0
$$

where the convection velocities $\mathbf{v}_{j}$ are prescribed in terms of stream-functions $\phi_{j}$ by $\mathbf{v}_{j}=\hat{\mathbf{z}} \times \nabla \phi_{j}$. Here the index $j$ takes the values $0,+$, and -. The fields $G_{j}$ are Lagrangian invariants given by $G_{0}=n_{i}$ and $G_{ \pm}=\psi+d_{e}^{2} J \pm d_{e} \rho_{s} n_{e}$, with $J=-\nabla^{2} \psi$, and the corresponding advecting stream-functions are $\phi_{0}=\Phi$ and $\phi_{ \pm}=\phi \pm \rho_{s} \psi / d_{e}$.

The Lagrangian representation of the dynamics leads to the "phase-mixing" picture of collisionless reconnection presented in Ref. [2]. According to this picture, the reconnection is enabled by the mixing of the invariants in a way analogous to the mixing of the distribution function during Landau damping. As a result of this mixing, the $G_{ \pm}$fields develop increasingly fine structure, like cream stirred in a cup of coffee. In order to determine the smoothness of the original fields, such as $n_{e}$ and $\psi$, we need to solve for these fields in terms of the invariants.

$$
\begin{aligned}
\psi & =\frac{1}{2}\left(1-d_{e}^{2} \nabla^{2}\right)^{-1}\left(G_{+}+G_{-}\right) \\
n_{e} & =\frac{1}{2}\left(G_{+}-G_{-}\right) / d_{e} \rho_{s} .
\end{aligned}
$$

The first of these expressions shows that $\psi$ is a smoothed version of the mixed-up $G_{ \pm}$fields, the inverse KelvinHelmholtz operator $\left(1-d_{e}^{2} \nabla^{2}\right)^{-1}$ having the effect of suppressing all scales below $d_{e}$. The second expression, Eq. (14), by contrast, shows that $n_{e}$ is not smoothed at all, and will contain all the fine scales produced by the mixing of 
the $G_{ \pm}$. Applying the $\nabla^{2}$ operator to Eq. (13) shows that the axial current $J$, like $n_{e}$, retains the fine structure of the $G_{ \pm}$. The dark and light structures (six of each, see Fig. 1 , case with $\rho_{s}=0.4$ ) in $n_{e}$ lying on and inside the separatrix can be recognized as being part of two spirals resulting from the roll-up of the invariants of the system in the counterclockwise and clockwise sense, respectively. $[2,23]$ These spirals represent a historical record of the reconnection up to that point, much like geological strata preserve the record of tectonic subduction and buckling.

Returning to the question of the behavior of the electrostatic potential, inspection of Eq. (8) shows that $\phi$ will itself only be smoothed when $\rho_{i}=0$. For $\rho_{i}>0$, by contrast, $\phi$ contains an unfiltered contribution from $n_{e}$ that exhibits progressively finer structure as the reconnection progresses. That is, $\phi$ as well as $n_{e}$ and $J$, exhibit a cascade towards smaller scales. It is easy to see that the fine structure is retained by the electron perpendicular velocity, since $\mathbf{v}_{e}=\hat{\mathbf{z}} \times \nabla\left(\phi-\rho_{s}^{2} n_{e}\right)$. Thus, the gyrofluid model describes the formation of electron beams much like the ones observed in kinetic simulations. $[16,17,31]$ The width of the beams is determined by the stretching and folding of the $G_{ \pm}$, and their parallel velocity is determined by the electron momentum conservation in the $\hat{\mathbf{z}}$ direction.

Note also that if, on the other hand, one considers the limit $\rho_{s} \rightarrow 0$, then $G_{ \pm} \sim \psi+d_{e}^{2} J$ and $\phi_{ \pm} \sim \phi$. Consequently, for small $\rho_{s}$ one expects not to see the stretching in opposite directions of $G_{+}$and $G_{-}$under the action of $\phi_{ \pm}$, because the magnetic contribution to the advection velocity fields $\mathbf{v}_{ \pm}$, which is what causes the opposite circulation of the two flows, gets suppressed. This can be seen in Fig.1, when comparing the two plots of $G_{+}$. In the case with small $\rho_{s}$ one does not see the spiral arms that are visible in the case with large $\rho_{s}$ and which are a result of the stretching in the clockwise sense caused by $\phi_{+}$(the case of $G_{-}$would be identical but with a spiral winding up anticlockwise [1]). Therefore, in spite of the similar features in the electron density around the separatrices, the two cases differ considerably in the structure of the underlying invariants and of the corresponding mixing process.

The Fourier representation offers an alternative explanation for the behavior of $\phi$ and the gyro-averaged potential $\Phi$. Let us write the periodic fields in Fourier series, so that $n_{e}=\sum_{\mathbf{k}=-\infty}^{+\infty} n_{e_{\mathbf{k}}}(t) \exp (i \mathbf{k} \cdot \mathbf{x})$ and analogously for the other fields. From (4) one obtains

$$
\begin{array}{r}
\phi_{\mathbf{k}}=\frac{1+\rho_{i}^{2} k^{2}}{k^{2}\left(1+\frac{\rho_{i}^{2}}{2} k^{2}\right)} n_{i_{\mathbf{k}}}-\frac{1+\rho_{i}^{2} k^{2}}{k^{2}} n_{e_{\mathbf{k}}}, \\
\Phi_{\mathbf{k}}=\frac{1+\rho_{i}^{2} k^{2}}{k^{2}\left(1+\frac{\rho_{i}^{2}}{2} k^{2}\right)^{2}} n_{i_{\mathbf{k}}}-\frac{1+\rho_{i}^{2} k^{2}}{k^{2}\left(1+\frac{\rho_{i}^{2}}{2} k^{2}\right)} n_{e_{\mathbf{k}} .}
\end{array}
$$


From these relations we deduce that for $\rho_{i} \rightarrow 0$,

$$
\phi_{\mathbf{k}} \sim \Phi_{\mathbf{k}} \sim\left(n_{i_{\mathbf{k}}}-n_{e_{\mathbf{k}}}\right) / k^{2}
$$

In this case, both $\phi$ and $\Phi$ are smoothed with respect to $n_{e}$, which is proportional to the sum of the convected fields $G_{+}$and $G_{-}$, and with respect to $n_{i}$ which is itself a convected field.

For $\rho_{i} \rightarrow+\infty$, by contrast,

$$
\begin{gathered}
\phi_{\mathbf{k}} \sim-\rho_{i}^{2} n_{e_{\mathbf{k}}} \\
\Phi_{\mathbf{k}} \sim-\frac{2}{k^{2}} n_{e_{\mathbf{k}}} .
\end{gathered}
$$

Thus we can see that, as $\rho_{i}$ increases, $\phi$ tends to become proportional to $n_{e}$ whereas $\Phi$ remains screened, an effect of the gyroaveraging. The similarity in the structure of $n_{e}$ and $\phi$ is indeed what Fig. 1 shows. Finally note that the above argument, based on the quasi-neutrality condition, is independent of the value of $\rho_{s}$ and holds also in the presence of nonuniform background density, in which case the perturbations to $n_{i}$, that were neglected in (9)-(10), must be retained. Another effect related to the presence of $\rho_{i}$ is the flattening of the electron density as far as the central region of the island is considered. This effect can be explained in terms of the different behavior of the velocity fields $\mathbf{v}_{ \pm}=\hat{\mathbf{z}} \times \nabla \phi_{ \pm}$which advect the Lagrangian invariants $G_{ \pm}$. Indeed, as far as $\rho_{i}$ increases we observe progressively weaker velocity fields inside the island, as shown in fig. 4 (first column plots) for two different values of $\rho_{i}(0.01,0.4)$ at the same value of $\rho_{s}=0.4$. Hence, the $G_{ \pm}$are less strechted and filamented far from the separatrices and tend to coincide inside the island, as shown for a particular $y$-chord in the second column of fig. 4. Here we note that peaks, which occur where the velocity field is stronger, develop well inside the island for small $\rho_{i}$, while they tend to be localized around the separatrices when $\rho_{i}$ increases. Since the electron density is recovered from the difference between the $G_{ \pm}$fields through relation (14), it follows that its profile tends to become flatter and flatter as we approach the $O$-point of the magnetic island.

\section{ENERGY CONSIDERATIONS}

The 3-field model (1)-(4) possesses the following conserved energy integral (Hamiltonian) [12]:

$$
H=\frac{1}{2} \int d^{2} x\left[|\nabla \psi|^{2}+d_{e}^{2}\left(\nabla^{2} \psi\right)^{2}+\rho_{s}^{2} n_{e}^{2}+\Phi n_{i}-\phi n_{e}\right]
$$

The various terms in $(20)$ represent the magnetic energy $\left(E_{B}\right)$, the parallel electron kinetic energy $\left(E_{k e}\right)$, the electron thermal energy $\left(E_{t h e}\right)$, the ion electrostatic energy $\left(E_{\text {eli }}\right)$ and the electron electrostatic energy $\left(E_{\text {ele }}\right)$, respectively. 
We consider the temporal evolution of the different contributions to the total energy for $\rho_{s}=0.4, \rho_{i}=0$ and $\rho_{s}=0$, $\rho_{i}=0.4$. First we note that, with our choice of equilibria and initial perturbations, the total energy (corresponding to its value at $t=0)$ is given by

$$
H=\frac{1}{2} \int d^{2} x\left[\left|\nabla \psi_{e q}(x)\right|^{2}+d_{e}^{2}\left(\nabla^{2} \psi_{e q}(x)\right)^{2}+\tilde{\Phi}(x, y) \tilde{n}_{i}(x, y)\right]+\frac{1}{2} \rho_{s}^{2} 4 \pi n_{0}^{2},
$$

where tildes denote the initial perturbations. The amount of energy turns out to be essentially the same for both cases, because the difference between the cases $\rho_{i}=0$ and $\rho_{i}=0.4$, present in the ion electrostatic energy, is less than $10^{-5}$ and because the presence of the constant term in (21) depending on $n_{0}^{2}$ is irrelevant for the investigation of the energy deviations from their initial values. By comparing the two plots of Fig. 5 we can see that, although the total energy is the same in both cases, the distribution into the various forms is quite different. In both cases we decided to consider the simulations reliable only when the loss of total energy due to numerical dissipation is less than $1 \%$. If we consider the plot for $\rho_{i}=0$ at $t=19$ and the plot for $\rho_{s}=0$ at $t=16$ (corresponding to magnetic islands of the same size) we see that essentially the same amount of magnetic energy has been lost in the two cases. This loss is, however, compensated in two different ways. For the cold ion case the reconnection process converts magnetic energy mainly into thermal electron energy (which prevails as $\rho_{s}$ is increased) and electron electrostatic energy. A smaller portion of magnetic energy also goes into parallel kinetic energy, although the latter starts to decrease when the total energy starts to be no longer conserved. We observe that in the cold ion limit the Hamiltonian can be written as

$$
H=\frac{1}{2} \int d^{2} x\left[|\nabla \psi|^{2}+d_{e}^{2}\left(\nabla^{2} \psi\right)^{2}+\rho_{s}^{2}\left(\nabla^{2} \phi\right)^{2}+|\nabla \phi|^{2}\right]
$$

Because in this limit $n_{e}=n_{i}+\nabla^{2} \phi$ the ion and electron electrostatic energy can be combined into a single electrostatic energy term which is also proportional to the perpendicular kinetic energy due to the $\mathbf{E} \times \mathbf{B}$ flow. The conversion can then of course be interpreted also as partial transformation into perpendicular kinetic energy.

In the opposite limit $\left(\rho_{s}=0, \rho_{i} \rightarrow+\infty\right)$, the Hamiltonian becomes

$$
H \sim \frac{1}{2} \int d^{2} x\left[|\nabla \psi|^{2}+d_{e}^{2}\left(\nabla^{2} \psi\right)^{2}+n_{i} \Phi+\frac{\rho_{i}^{2}}{4}\left(\nabla^{2} \Phi\right)^{2}\right] .
$$

In this limit the electron thermal energy vanishes and almost all the magnetic energy is converted into electron electrostatic energy, which in this limit becomes proportional to the square of the laplacian of the gyroaveraged potential. In particular, if one assumes that $\psi=O(1)$ and $n_{e}=O(1)$ as $\rho_{i} \rightarrow+\infty$, then $\phi=O\left(\rho_{i}^{2}\right)$, and the electron electrostatic will tend to grow and dominate the other terms in that limit. We note also that, compared to the cold-ion 
case, the presence of finite ion temperature determines a decrease in the parallel kinetic energy as shown in Fig. 6, where a comparison between two cases with $\rho_{s}=0.2$ and $\rho_{i}=0,0.2$, respectively, is considered.

In the limit of negligible $n_{i}$ we can recover the following expressions:

$$
\begin{aligned}
\frac{d}{d t} \frac{1}{2} \int d^{2} x\left(|\nabla \psi|^{2}+d_{e}^{2}\left(\nabla^{2} \psi\right)^{2}\right) & =\int d^{2} x \nabla^{2} \psi\left[\nabla^{-2} n_{e}, \psi\right]+\left(\rho_{i}^{2}+\rho_{s}^{2}\right) \int d^{2} x \nabla^{2} \psi\left[\psi, n_{e}\right], \\
\frac{d}{d t} \frac{\rho_{s}^{2}}{2} \int d^{2} x n_{e}^{2} & =-\rho_{s}^{2} \int d^{2} x \nabla^{2} \psi\left[\psi, n_{e}\right] \\
-\frac{d}{d t} \frac{1}{2} \int d^{2} x \phi n_{e} & =-\int d^{2} x \nabla^{2} \psi\left[\nabla^{-2} n_{e}, \psi\right]-\rho_{i}^{2} \int d^{2} x \nabla^{2} \psi\left[\psi, n_{e}\right] .
\end{aligned}
$$

These relations show how the sum of the magnetic and parallel kinetic energy, which is essentially the only form of energy available at the initial state, is transferred into different channels. Electron temperature terms are a source for electron thermal energy, whereas the corresponding ion temperature term and the convective term on the right-hand side of (24) provide a source for the electron electrostatic energy.

\section{CONCLUSION}

We have shown that, in some respects, ion gyration (parametrized by $\rho_{i}$ ) plays a role similar to electron parallel compressibility (parametrized by $\rho_{s}$ ). Both give rise to an acceleration of reconnection compared to the case of cold plasma $\left(\rho_{s}=\rho_{i}=0\right)$, and both have the effect of stabilizing the Kelvin-Helmholtz instabilities that have been observed in the collisionless reconnection of cold plasma.[4, 5, 21, 22] In both cases, also, the acceleration of reconnection is consistent with changes in the dispersion relation, fast reconnection occurring when the dispersion curve has a convex segment. [26] Lastly, when $\rho_{s}$ is finite, also in the presence of finite ion temperature, the acceleration observed in hot plasma can be interpreted as resulting from the roll-up of the two Lagrangian invariants $G_{ \pm}$by the convection cells associated with the reconnection. Such roll-up is energetically favorable since the magnetic energy is given by the integral of $|\nabla \psi|^{2}$, and $\psi$ is determined from the difference of the $G_{ \pm}$by a smoothing operation. The smoothing filters the short scales, so that the magnetic energy in the rolled-up state is reduced from that in the initial state [2]. Beyond their superficial similarities, however, the cases of cold and hot ions exhibit important differences. Whereas the electrostatic potential is smooth in the case of cold ions, for hot ions it retains the fine structures created by the roll-up of the $G_{ \pm}$. As a result, the finite- $\rho_{i}$ model exhibits electron beams similar to those observed in particle simulations. [16-18, 31] This feature is important for the study of the particle energization occurring during magnetic reconnection. Moreover, another feature of finite ion temperature is a flattening of the electron density inside the 
magnetic island. This can be explained in terms of a decreasing of the speed of $\mathbf{v}_{ \pm}$going from the separatrices to the inner region of the island. Also the energy distribution is affected by the presence of hot ions. Indeed, when finite ion temperature is taken into account a relative increase in the electron electrostatic energy is compensated by a decrease in the parallel kinetic and electron thermal energies.

Lastly, we note that while particle codes are highly effective for small guide fields, their advantages recede for large guide fields and for current systems of macroscopic size. For such problems, the present paper shows that gyrofluid codes may prove to be valuable research tools. A good understanding of gyrofluid dynamics may also prove useful in the development of implicit moment algorithms [32] for gyrokinetic codes, since the gyrofluid equations are given by the moments of the gyrokinetic equation [33].

\section{Acknowledgments}

The authors acknowledge fruitful discussions with Dario Borgogno. This work was supported by the European Community under the contracts of Association between EURATOM and ENEA and between EURATOM, CEA, and the French Research Federation for fusion studies. FW was supported in part by a fellowship from the Katholieke Universiteit Leuven and by the US Department of Energy under contract DE-FG05-96ER-54346. The views and opinions expressed herein do not necessarily reflect those of the European Commission. Financial support was also received from the Agence Nationale de la Recherche (ANR EGYPT).

[1] E. Cafaro, D. Grasso, F. Pegoraro, F. Porcelli, and A. Saluzzi, Phys. Rev. Lett. 80, 4430 (1998).

[2] D. Grasso, F. Califano, F. Pegoraro, and F. Porcelli, Phys. Rev. Lett. 86, 5051 (2001).

[3] B. Scott and F. Porcelli, Phys. Plasmas 11, 5468 (2004).

[4] D. Del Sarto, F. Califano, and F. Pegoraro, Modern Phys. Lett. B 20, 931 (2006).

[5] D. Grasso, D. Borgogno, and F. Pegoraro, Phys. Plasmas 14, 055703 (2007).

[6] E. Tassi, P. Morrison, D. Grasso, and F. Pegoraro, Nucl. Fusion 50, 034007 (2010).

[7] E. Tassi, P. J. Morrison, F. L. Waelbroeck, and D. Grasso, Plasma Phys. and Control. Fusion 50, 085014 (2008).

[8] A. Brizard, Phys. Fluids B 4, 1213 (1992).

[9] T. J. Schep, F. Pegoraro, and B. N. Kuvshinov, Phys. Plasmas 1, 2843 (1994).

[10] B. N. Kuvshinov, F. Pegoraro, and T. J. Schep, Phys. Lett. A 191, 296 (1994).

[11] D. Strintzi, B. D. Scott, and A. J. Brizard, Phys. Plasmas 12, 052517 (2005).

[12] F. L. Waelbroeck, R. D. Hazeltine, and P. J. Morrison, Phys. Plasmas 16, 032109 (2009).

[13] D. Grasso, F. Califano, F. Pegoraro, and F. Porcelli, Plasma Physics Reports 26, 512 (2000).

[14] N. F. Loureiro and G. W. Hammett, J. Comp. Phys. 227, 4518 (2008).

[15] N. H. Bian and E. P. Kontar, Physics of Plasmas 17, 062308 (2010).

[16] J. F. Drake, M. A. Shay, W. Thongthai, and M. Swisdak, Phys. Rev. Lett. 94, 095001 (2005).

[17] P. L. Pritchett, J. Geophys. Res. 111, A10212 (2006).

[18] J. F. Drake, P. A. Cassak, M. A. Shay, M. Swisdak, and E. Quataert, The Astrophysical Journal Letters 700, L16 (2009).

[19] G. Fiksel, A. F. Almagri, B. E. Chapman, V. V. Mirnov, Y. Ren, J. S. Sarff, and P. W. Terry, Phys. Rev. Lett. 103, 145002 (2009).

[20] J. Egedal, W. Daughton, J. F. Drake, N. Katz, and A. Lê, Phys. Plasmas 16, 050701 (2009).

[21] D. Del Sarto, F. Califano, and F. Pegoraro, Phys. Rev. Lett. 91, 235001 (2003). 
[22] D. D. Sarto, F. Califano, and F. Pegoraro, Phys. Plasmas 12, 012317 (2005).

[23] D. Grasso, F. Califano, F. Pegoraro, and F. Porcelli, Plasma Phys. and Control. Fusion 41, 1497 (1999).

[24] S. Vincena, W. Gekelman, and J. Maggs, Phys. Rev. Lett. 93, 105003 (2004).

[25] X. yu Wang, X. yi Wang, Z. xing Liu, and Z. yuan Li, Phys. Plasmas 5, 4395 (1998).

[26] B. N. Rogers, R. E. Denton, J. F. Drake, and M. A. Shay, Phys. Rev. Lett. 87, 195004 (2001).

[27] D. Grasso, L. Margheriti, F. Porcelli, and C. Tebaldi, Plasma Phys. and Control. Fusion 48, L87 (2006).

[28] F. Porcelli, Phys. Rev. Lett. 66, 425 (1991).

[29] J. Birn, J. Drake, M. Shay, B. Rogers, R. Denton, M. Hesse, M. Kuznetsova, Z. Ma, A. Bhattacharjee, A. Otto, et al., J. Geophys. Res.-Space Phys. 106, 3715 (2001).

[30] L. Chacón, A. N. Simakov, V. S. Lukin, and A. Zocco, Phys. Rev. Lett. 101, 025003 (2008).

[31] P. L. Pritchett, Phys. Plasmas 12, 062301 (2005).

[32] G. Lapenta, J. U. Brackbill, and P. Ricci, Phys. Plasmas 13, 055904 (2006).

[33] T. S. Hahm, W. W. Lee, and A. Brizard, Phys. Fluids 31, 1940 (1988). 

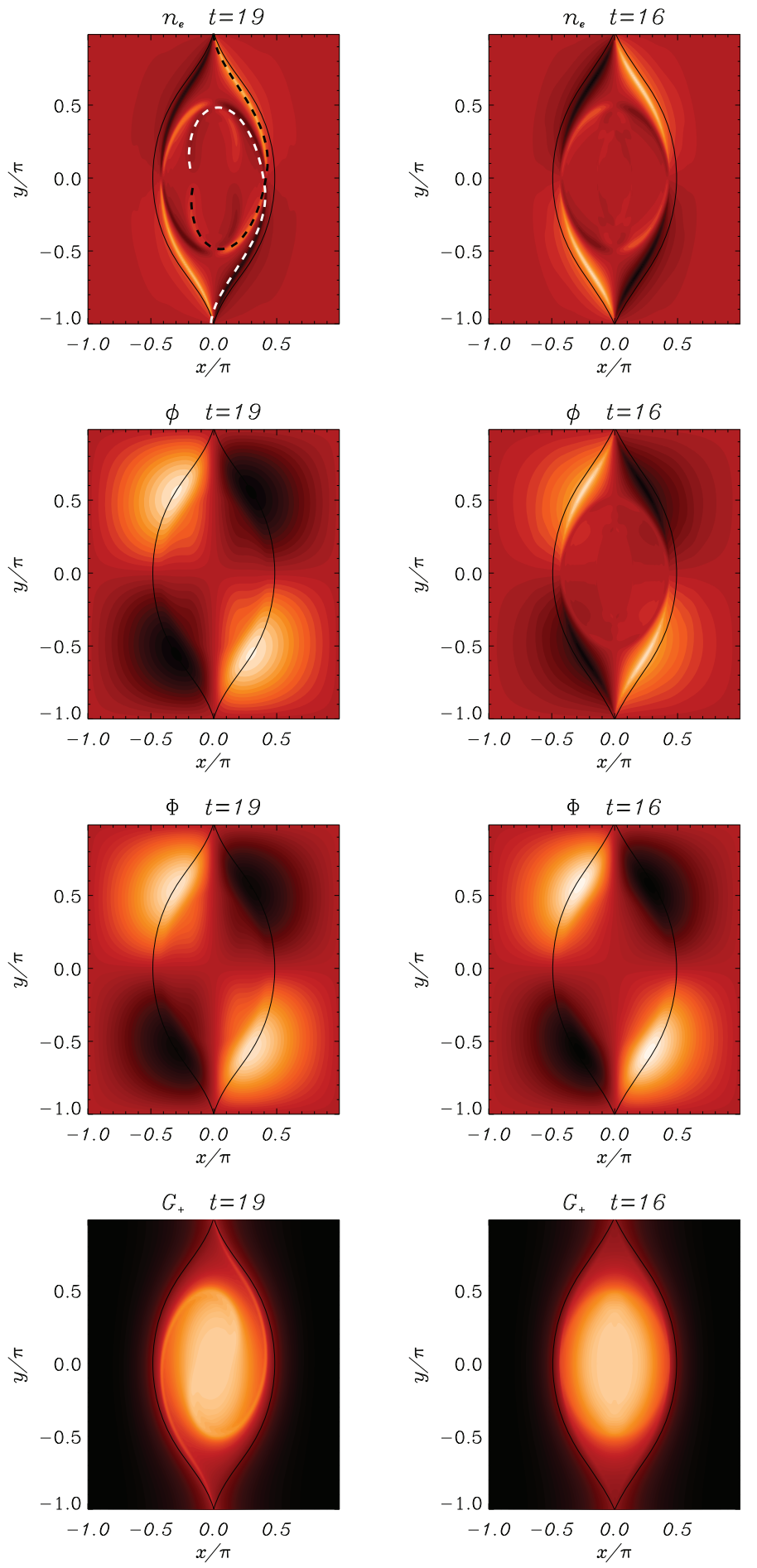

FIG. 1: (Color online). Contour plots of electron density $n_{e}$, electrostatic potential $\phi$, gyroaveraged electrostatic potential $\Phi$ and the Lagrangian invariant $G_{+}$for $\rho_{s}=0.4, \rho_{i}=0.01$ (left column) and $\rho_{s}=0.01, \rho_{i}=0.4$ (right column). Contour plot of the magnetic island at the corresponding time have been superimposed onto each plot. The color scale goes from black (minimum value) to white (maximum value). In the plot of $n_{e}$ for $\rho_{s}=0.4, \rho_{i}=0.01$ dashed black and white lines are superimposed in order to show the presence of the two spirals resulting from the roll-up of the $G_{ \pm}$invariants. The black (white) spiral arm connect regions of high (low) electron density. The value of the electron skin depth is $d_{e}=0.2$. 

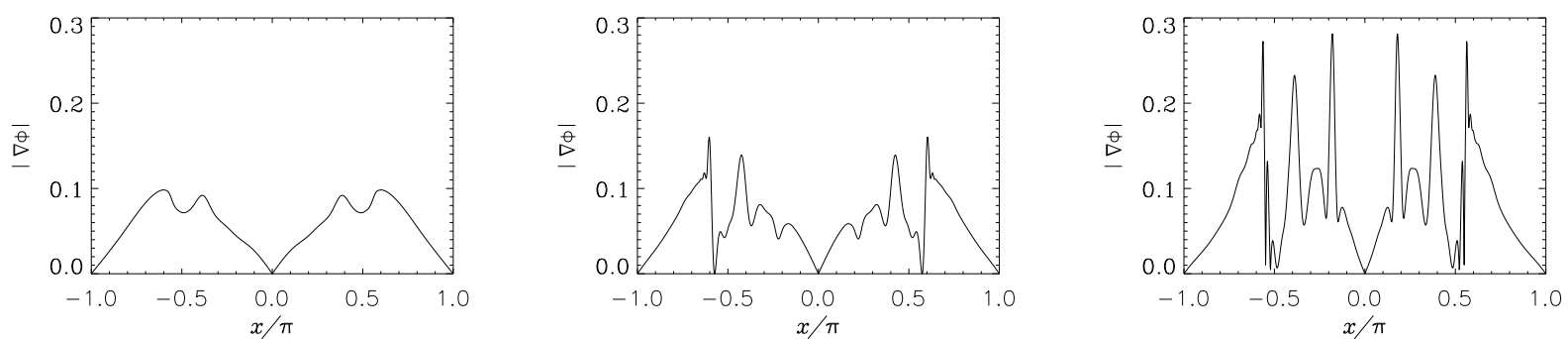

FIG. 2: Plots of $|\nabla \phi|$, at $y / \pi=-1 / 2$, as a function of $x / \pi$, for $\rho_{s}=0.4, d_{e}=0.2$ and for three different values of $\rho_{i}$. The plots on the left-hand side, in the middle and on the right-hand side correspond to $\rho_{i}=0, \rho_{i}=0.2$ and $\rho_{i}=0.4$, respectively. It is possible to observe that, as $\rho_{i}$ increases, the amplitude of the peaks becomes larger and steeper gradients form in the perpendicular electric field. At the times which the three plots refer to, the corresponding magnetic islands have reached the same width.

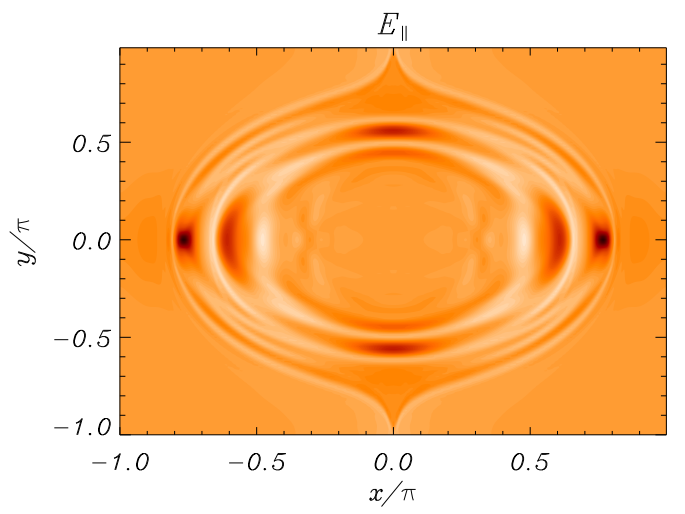

FIG. 3: (Color online). Contour plot of the parallel electric field $E_{\|}=-\partial \psi / \partial t-[\phi, \psi]$ for $\rho_{s}=0.4, \rho_{i}=0.4$ and $d_{e}=0.2$. The plot exhibits fine structures which are determined by the filamentation of $\phi$ that occurs as $\rho_{i}$ increases. 

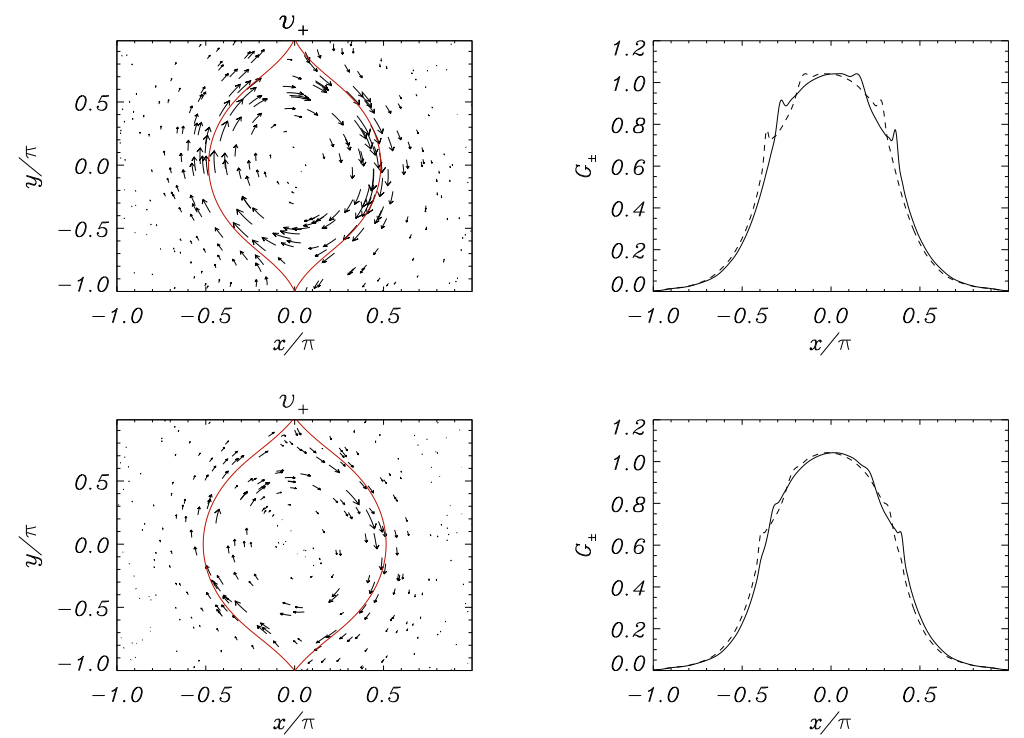

FIG. 4: (Color online). Plots of velocity field $\mathbf{v}_{+}$and profiles of $G_{+}$(dashed line) and $G_{-}$(solid line) at $y / \pi=-1 / 3$ for $\rho_{i}=0.01$ (top row) and $\rho_{i}=0.4$ (bottom row). In both cases $\rho_{s}=0.4$ and $d_{e}=0.2$. By comparing the plots of the velocity fields one observes that for small $\rho_{i}$ there exist high speed regions also inside the island (overplotted in red). This favours the formation of regions in which the difference between the local values of $G_{+}$and $G_{-}$is enhanced. For large $\rho_{i}$, on the other hand, the velocity field is weaker inside the island, with respect to the separatrix regions. As a consequence, $G_{+}$and $G_{-}$tend to coincide inside the island and therefore the electron density is locally flattened.
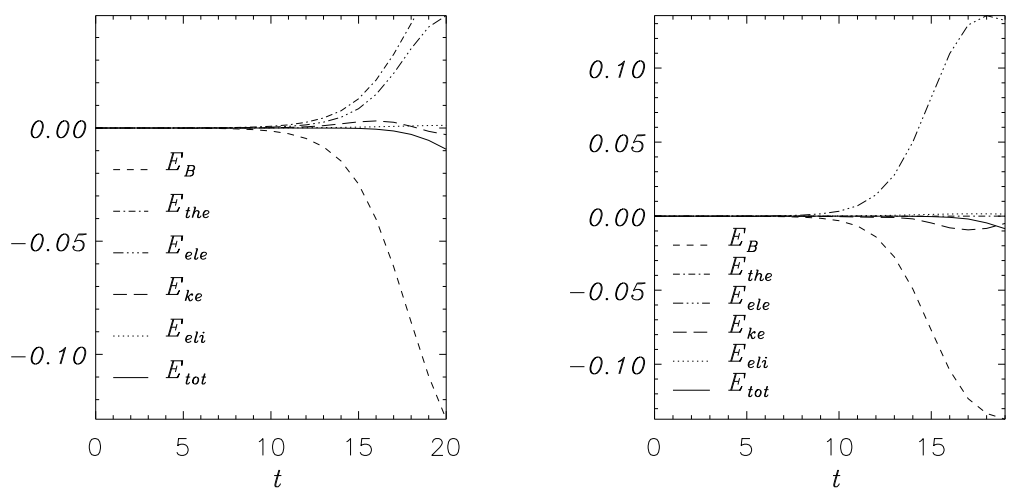

FIG. 5: Temporal evolution of energy deviations from the initial values, according to eqs. (20) and $(21)$, for $\rho_{s}=0.4, \rho_{i}=0$ (left) and $\rho_{s}=0, \rho_{i}=0.4$ (right). In both cases $d_{e}=0.2$. 

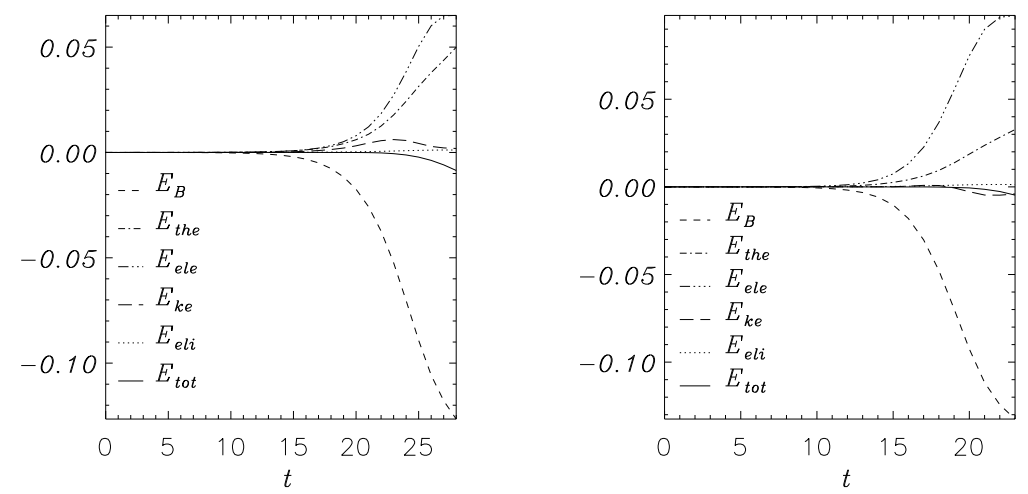

FIG. 6: Temporal evolution of energy deviations from the initial values, according to eqs. (20) and $(21)$, for $\rho_{s}=0.2, \rho_{i}=0$ (left) and $\rho_{s}=0.2, \rho_{i}=0.2$ (right). In both cases $d_{e}=0.2$. 